\title{
Numerical and Experimental Study of Electromagnetically Driven Vortical Flows
}

\author{
S. Kenjereš ${ }^{1}$, J. Verdoold, M. J. Tummers, K. Hanjalić and C. R. Kleijn \\ Department of Multi-Scale Physics, Faculty of Applied Sciences and J. M. Burgerscentre for Fluid \\ Dynamics, Delft University of Technology, \\ Prins Bernhardlaan 6, 2628 BW Delft, The Netherlands
}

\begin{abstract}
The paper reports on numerical and experimental investigations of electromagnetically driven vortical flows of an electrically conductive fluid in a generic setup. Two different configurations of permanent magnets are considered: a 3-magnet configuration in which the resulting Lorentz force is focused in the wall-boundary layers, and a 2-magnet configuration which creates a centrally located intensive swirling motion. For both configurations the intensity of the Lorentz force could be varied by variation of the electrode DC current between $0.5 \mathrm{~A}$ and $10 \mathrm{~A}$.

A comparative assessment of measured (PIV) and numerically calculated (LES with electromagnetically extended subgrid closure) velocity fields showed good agreement for both configurations. It is demonstrated that the newly designed setup can be used for fundamental studies of the interactions between fluid flow, turbulence and electromagnetic fields and provide detailed insights into the underlying physics of these interactions. This in turn can be used to optimise magnetic control of flow, turbulence and heat transfer in various configurations of practical relevance.
\end{abstract}

\section{Keywords}

flow control, Lorentz force, magnetic and electric fields, vortical flows

\footnotetext{
${ }^{1}$ Corresponding author. Tel.::+31-15-2783649; Fax.+31-15-2782838. E-mail address: S.Kenjeres@tudelft.nl
} 


\section{Introduction}

The accurate predictions of fluid flow, heat and mass transfer in electrically conductive fluids subjected to electromagnetic fields is an important prerequisite for design, optimization and control of many technological processes. Examples include continuous steel casting, electromagnetic mixing and stirring in metallurgy, arc-welding, crystal growth and aluminium cells. These applications involve complex interactions between fluid flow, heat transfer, turbulence and electromagnetic fields, which are notoriously difficult to determine experimentally because of the inaccessibility of most liquids involved to common experimental techniques, and because of the need to measure simultaneously fluid velocity, temperature, magnetic and electric fields.

In order to provide a better insight into these interactions, we performed combined numerical and experimental studies in simplified configurations where many of these physical phenomena can be well controlled and studied in detail. Fig. 1 shows the generic experimental set up. It consists of a rectangular tank where the electromagnetic forcing is imposed by interactions between the electric field generated by two electrodes in the upper part of the side walls, and the magnetic field imposed by different combinations and orientations of permanent magnets under the bottom wall.

The permanent magnets have dimensions $0.084 \times 0.040 \times 0.040 \mathrm{~m}^{3}$ and are arranged in arrays of alternating polarity. The distance between each magnet is $0.040 \mathrm{~m}$, resulting in a maximum magnetic field strength of $1.0 \mathrm{~T}$ inside the tank. For the present investigation, two configurations have been studied, a 2 magnetic block configuration with a North and South polarity and a 3 magnetic block configuration using a North-South-North block layout. The electric field is generated by supplying DC current to electrodes mounted in the upper corner of two opposing side walls, see Fig. 1. The controllable DC current could be varied be tween $0.5 \mathrm{~A}$ and $10 \mathrm{~A}$. Due to the relatively large electrical current, induction effects can be neglected and Ohm's law can be approximated by $\mathbf{J}=\sigma \mathbf{E}$. Then, the resulting Lorentz force for the present range of experiments can be expressed as $\mathbf{F}^{\mathbf{L}}=\mathbf{E} \times \mathbf{B}$, which is constant in time. Water with $7 \% \mathrm{Na}_{2} \mathrm{SO}_{4}$ electrolyte solution is used as a working fluid, which enhances the fluid's electric conductivity $\sigma$ to $5.5 \mathrm{~S} \mathrm{~m}^{-1}$. The upper and lower plate can be kept at different temperatures, making it possible to study effects of the electromagnetic forcing on the local heat transfer, Hanjalić and Kenjereš (2000,2001,2006), Kenjereš and Hanjalić (2004), Verdoold et al. (2006b). The intensity of the flow forcing can be easily controlled by changing the intensity of the electrical current supplied to the electrodes. In the present study we consider the isothermal situation only. 
This investigation represents a continuation of the previous research of Verdoold et al. $(2003,2005)$ where initial LIF flow visualisations were performed, but without velocity measurements. In contrast to the work of Cardoso et al. (1994), Hennoch and Stace (1995) and Rossi et al. (2006a, 2006b) where arrays of alternating magnetic blocks are used which result in typical two-dimensional laminar flow features, here we focus on genuinely three-dimensional flow patterns in transitional and fully developed turbulent regimes. Particle Image Velocimetry (PIV) data will be used for validation of the Large Eddy Simulations. This combination of experimental and numerical investigations will make it possible to provide a detailed physical insight into interactions between fluid flow and electromagnetic fields in the configurations considered.

\section{Equations and subgrid model}

For Large Eddy Simulations (LES), the flow of an electrically conductive fluid subjected to an external electromagnetic field can be described by the following equations, Shimomura (1991), Kenjereš and Hanjalić (2000, 2004):

$$
\begin{gathered}
\frac{\partial \overline{U_{i}}}{\partial t}+\overline{U_{j}} \frac{\partial \overline{U_{i}}}{\partial x_{j}}=\frac{\partial}{\partial x_{j}}\left(\nu \frac{\partial \overline{U_{i}}}{\partial x_{j}}-\tau_{i j}\right)-\frac{1}{\rho} \frac{\partial \bar{P}}{\partial x_{i}}+F_{i}^{L} \\
\tau_{i j}=\frac{1}{3} \tau_{k k} \delta_{i j}-\nu_{t} \overline{S_{i j}}, \\
F_{i}^{L}=\frac{1}{\rho} \epsilon_{i j k} \overline{E_{j}} \overline{B_{k}} \\
\nu_{t}=\nu_{s} \exp \left[-\left(\frac{\sigma}{\rho}\right)\left(C_{m} \Delta\right)^{2}\left|B_{0}\right|^{2} / \nu_{s}\right], \\
\nu_{s}=\left(C_{s} \Delta\right)^{2}\left(\overline{S_{i j}} \overline{S_{i j}}\right)^{1 / 2}, \overline{S_{i j}}=\frac{1}{2}\left(\frac{\partial \overline{U_{i}}}{\partial x_{j}}+\frac{\partial \overline{U_{j}}}{\partial x_{i}}\right), \\
\frac{\partial \overline{U_{i}}}{\partial x_{i}}=0, \frac{\partial \overline{B_{i}}}{\partial x_{i}}=0, \frac{\partial \overline{E_{i}}}{\partial x_{i}}=0
\end{gathered}
$$

$\bar{E}$ and $\bar{B}$ stand for the electric and magnetic field that are calculated from a simplified set of Maxwell's equations by applying Biot-Savart law for permanent magnets and electrodes, Akoun and Yonnet (1984). Note that the subgrid turbulent viscosity is calculated from the magnetically extended Smagorinsky model in order to account for additional magnetic reduction of velocity fluctuations, as proposed by Shimomura (1991). $B_{0}=\sqrt{B_{x}^{2}+B_{y}^{2}+B_{z}^{2}}$ is the magnitude of the imposed magnetic field that is constant in time but it varies for different locations inside of the tank, i.e. $B_{0}=B_{0}(x, y, z) . C_{S}=0.1$ and 
Table 1: Overview of some of simulated configurations. The magnetic field strength of permanent magnets was $\left|B_{0}\right|=1 \mathrm{~T}$, giving $H a=8$.

\begin{tabular}{|c|c|c|c|c|c|c|}
\hline & \multicolumn{2}{|c|}{$R e$} & \multicolumn{2}{c|}{$N_{M H D}$} & \multicolumn{2}{c|}{$N_{E M H D}$} \\
\hline \hline Applied DC current & $I=1 \mathrm{~A}$ & $I=10 \mathrm{~A}$ & $I=1 \mathrm{~A}$ & $I=10 \mathrm{~A}$ & $I=1 \mathrm{~A}$ & $I=10 \mathrm{~A}$ \\
\hline 2 Magnets & 1900 & 8250 & 0.035 & 0.008 & 1.3 & 0.7 \\
3 Magnets & 1100 & 4450 & 0.06 & 0.015 & 4. & 0.24 \\
\hline
\end{tabular}

$C_{m}=1.4$ are the model constants. It can be seen that this model reduces to the standard Smagorinsky model in the absence of a magnetic field.

A range of electric currents between 0.5 and 10 A has been considered. Depending on the imposed electromagnetic forcing, the generated flow can be laminar, transitional or fully developed turbulent, with Reynolds numbers (based on the distance between the upper and lower plate and on a maximum value of the long-time averaged induced velocity field) in the range of $500 \leq R e \leq 8250$, the larger values corresponding to the higher electric current, Table. 1. We defined two types of the interactive number $N$ (that represents a ratio between electromagnetic and inertial forces): a standard one based on the strength of the imposed magnetic field that ignores electric current contributions $\left(N_{M H D}\right)$, and a new one $\left(N_{E M H D}\right)$ - based on the total electromagnetic contributions (expressed through the imposed volumetric electromagnetic force, $f_{E M H D}$ ), Thibault and Rossi (2003). $N_{M H D}$, which accounts for forcing of the fluid solely through the action of the permanent magnets is low $\left(0.008 \leq N_{M H D} \leq 0.06\right)$, indicating very weak interactions since the electric conductivity of the working fluid is rather low. In contrast, the interactive number $N_{E M H D}$ based on total electromagnetic contributions from both the electrodes and the permanent magnets, has significantly higher values $\left(0.24 \leq N_{E M H D} \leq 4\right)$.

The used numerical mesh of $128 \times 128 \times 82 \mathrm{CVs}$, clustered in the proximity of bottom and top walls $\left(z_{n}^{+}=0.5\right)$ with a grid expansion factor below 1.2, resulted in well-resolved LES with a maximum ratio between turbulent and molecular viscosity below one.

\section{Numerical method}

The set of equations (1)-(6) is discretised by using a finite-volume Navier-Stokes/Maxwell solver for three-dimensional non-orthogonal geometries, Kenjereš et al. (2006), Kenjereš and Hanjalić (2007a, 
2007b), Kenjereš (2008). The Cartesian vector and tensor components for both fluid flow $\left(\overline{U_{i}}, \bar{P}\right)$ and electromagnetic variables $\left(\overline{B_{i}}, \overline{E_{i}}\right)$ are defined in the cell centres of a collocated mesh. The Rhie-Chow interpolation and SIMPLE algorithm are employed in order to prevent decoupling between velocity and pressure fields for such collocated meshes. In order to eliminate artifical numerical diffusion in the LES model, the second-order central differencing scheme (CDS) is used for discretisation of both diffusive and convective terms in the momentum equation. A fully implicit second-order time integration scheme based on 3-consecutive time steps is used for discretisation of time dependent terms. The typical time steps corresponded to CFL number of 0.5.

The total time integration covered an interval of $7200 \mathrm{~s}$ of real time. The numerical solver can be run on a single CPU or in parallel mode utilising the MPI domain decomposition directives. The simulations reported in this paper were performed by using 16-32 CPUs with almost ideal speed-up scaling.

\section{Experimental technique}

The flow has been experimentally investigated in a $600 \times 600 \times 155 \mathrm{~mm}^{3}$ glass enclosure, earlier described in Verdoold et al. (2006a, 2006b, 2008). In contrast to those buoyancy driven experiments, the top copper plate has been replaced by a glass window and permanent magnetic NdFeB blocks $\left(\left|B_{0}\right|=1.0 \mathrm{~T}\right)$ have been placed on an iron plate in the vicinity of the bottom wall. The iron plate enlarges the magnetic field line density inside the measurement volume and is necessary to mount the separate magnetic blocks. This bottom wall is $9 \mathrm{~mm}$ thick and made of copper, which will not influence the imposed magnetic field. Platinum wire electrodes are placed at two opposing side walls of the tank in designated electrode cavities to introduce the current density, as shown in Fig. 1. The current needed to force the system can be altered between 0 and $10 \mathrm{~A}$ at approximately $10 \mathrm{~V}$.

The velocity fields presented in this paper are measured by using a PIV system manufactured by ILA, which included a Continuum Minilite double-pulsed Nd:YAG laser with a pulse energy of $25 \mathrm{~mJ}$ with wavelength $\lambda=532 \mathrm{~nm}$. Neutrally buoyant hollow glass spheres (Potters-Ballotini) with a size in the order of $10 \mu \mathrm{m}$ are mixed in the working fluid in a concentration of approximately $1.0 \times 10^{-2} \mathrm{~g}^{-1}$. A PCO Sensicam camera with a scan area of $8.6 \times 6.9 \mathrm{~mm}$ and a resolution of $1280 \times 1024$ pixels was used to record the images, using a $55 \mathrm{~mm}$ lens with numerical aperture $f^{\#}=4$. Snapshots are captured at a frequency of $2 \mathrm{~Hz}$. The image snapshots are analysed in three subsequent steps using Gaussian local median filtering and interpolation for interrogation areas of $32 \times 32$ pixels in the first step reducing to 
$8 \times 8$ pixels with $50 \%$ overlap. With a magnification factor $M$ of $6 \mathrm{px} \mathrm{mm}^{-1}$, snapshot time difference $\Delta t$ of $30 \mathrm{~ms}$, the in-plane and out-of-plane loss of correlation criteria for a valid PIV analysis by Keane and Adrian (1990) have been successfully met. After filtering at least $96 \%$ of the resulting vectors was valid. For statistical analysis about 5000 frames are processed per configuration.

The PIV technique has been used to provide velocity data in $(x, z)$ planes in the middle of the tank, normal to both walls, as well as in $(x, y)$ planes parallel to the walls. For the $(x, z)$ plane measurements, two cameras were used to generate two complementary images of the enclosure. The full view is constructed using an interpolation algorithm for irregularly spaced data, following Shepard (1968). The new grid has a displacement $d$ of $5 \mathrm{~mm}$ between the datapoints in both directions. The value of the new datapoint was derived from a distance weighted average of the old grid points in a circle with radius $r$, where $r=d$ was chosen as disc size. This resulted in approximately 6 to 10 points from the old grid defining one point in the new grid within the distance $r$ and approximately 3 points within a distance $r / 3$. The effective area of view for the $(x, y)$ plane is $0.2 \times 0.3 \mathrm{~m}^{2}$, while the $(x, z)$ planes comprises the full tank, i.e. $0.6 \times 0.155 \mathrm{~m}^{2}$. For both cases, the post-processing procedure resulted in a vector field with a resolution of $0.5 \times 0.5 \mathrm{~cm}^{2}$.

\section{Results}

The practical feature of the designed setup is that different electromagnetic forcing can be imposed by simple reconfiguration of the permanent magnets and their orientation with respect to the fixed DC electrodes of opposite polarity. As such, it can be used for testing different scenarios with locally imposed non-homogenous body forces and their potential for flow and heat transfer control. In Figs. 2 and 3 we show computational results for two characteristic flow patterns originating from a configuration with two-magnets (shear forcing) and a configuration with three-magnets (pump-in-pump-out). The first configuration provides a well-defined vortical tornado-like structure, with a distinct low-pressure region in its centre, Fig. 2. The second configuration results in a local wall-jet motion upward and then downward forcing in the vertical plane above the underlying magnets, Fig. 3.

A comparison between PIV and LES (in the central vertical plane, $y=0$ ) for the 3-magnets situation is shown in Fig. 4. Both velocity vectors and contours of the horizontal velocity component are compared. This configuration leads to relatively low velocities, since the applied current is rather low $(I=0.5 \mathrm{~A})$. Despite relatively weak forcing, the resulting flow pattern exhibits complex behaviour. It can be seen 
that the LES results closely mimic the measured PIV fields - including the vertical upward motion in the proximity of the central magnet, as well as the wall-jet like patterns before and after the first and third magnet. A significant deviation is observed only in the upper left corner, where the PIV results show a more pronounced recirculation compared to the LES results. The horizontal velocity profiles at different vertical locations in the vertical midplane $(y=0 \mathrm{~m})$ are shown in Fig. 5. Positive values of the horizontal velocity (indicating a recirculating flow pattern) are visible at the $z=0.01$ and $0.02 \mathrm{~m}$ locations. The agreement between LES and PIV is good for all considered profiles - the dynamical adjustment of flow forcing with the wall-distance is nicely captured. Some deviation between PIV and LES is visible in the proximity of the left wall, where the PIV results did not capture the exact near-wall behaviour. This is due to experimental errors caused by the light reflection at the wall and the subsequent field reconstruction.

The 2-magnets configuration generates well-defined swirling (tornado-like) flow patterns in the central part of the setup, see Fig. 6. Now a significantly higher electrical current is imposed ( $I=10$ A) resulting in a well-developed turbulent flow. In addition to the main central vortex, a pair of smaller secondary vortical structures is also present, see Fig. 6-above. The central vortical structure becomes fully symmetrical and the secondary vortices vanish as the distance from the lower wall increases (results not shown here). A comparison between time-averaged velocity fields from PIV and LES in the zoomed-in region denoted by a solid rectangle is shown in Fig. 6-below. It can be seen that both LES and PIV show the strong vortical structure with identical size and velocity magnitude. The PIV shows a slightly asymmetrical distribution, with slightly stronger downward motion at the left edge of the vortex and slightly weaker upward motion along the left vortex edge, while the LES provides perfectly symmetrical distributions. Horizontal velocity magnitude profiles, circumferentially averaged (from the centre towards the outer edge of the vortex) are shown in Fig. 7. It can be seen that the peak value stays constant with wall distance, whereas its location gradually moves towards the outer edge of the vortex. Again, good agreement between PIV and LES is obtained, especially with respect to the exact peak value, while some discrepancies are visible at locations towards the vortex edge. The time-averaged vertical profiles of the horizontal velocity at two different locations are shown in Fig. 8. Note that the measured vertical profiles do not span the entire height of the setup. The locations of the velocity peak values in the proximity of the lower wall are nicely captured at both locations. At $x=-0.05 \mathrm{~m}$ some deviations between PIV and LES can be observed in the upper part for $z>0.075 \mathrm{~m}$. The velocity peak value is slightly underestimated at $x=0.05 \mathrm{~m}$, but the qualitative shape of the profile is well captured.

In order to provide insights into the vortical structures generated by the Lorentz force, the vertical vorticity component contours in two characteristic horizontal planes are shown in Figs. 9 and 10. The 
long term averaged fully developed LES velocity fields are used for calculation of the vorticity components.

The vertical vorticity for the 2-magnets configuration (with applied DC current of $I=10 \mathrm{~A}$ ) in the proximity of the bottom wall $(z=0.005 \mathrm{~m})$ portrays a relatively simple central pattern with three counter-rotating structures, Fig. 9-above. Such centrally located flow structures progressively affect a considerably larger area than simply the area just above the top surface of permanent magnets directly affected by the Lorentz force. This is due to inertial effects that effectively put working fluid in motion almost everywhere inside the tank. Through interactions of these central flow structures with the side walls, additional flow reorientation takes place in the proximity of the side walls. The flow structures in the central horizontal plane $(z=0.0775 \mathrm{~m})$ show a mini tornado like single swirling structure, similar to that found by Yih (2007), weakly interacting with the side walls, see Fig. 9-below.

The configuration with 3-magnets, despite a lower intensity of applied current $(I=0.5 \mathrm{~A})$, generates quite a complex flow pattern with three pairs of counter-rotating vortical structures in the central part of the setup for the horizontal plane in the proximity of bottom wall $(z=0.005 \mathrm{~m})$, Fig. 10-above. A strong horizontal wall-jet motion is created and strong vortical structures are also present in the sidewall impingement regions (the left wall of the setup). This horizontal jet imposes a significant vertical component of velocity along left side wall and imprints of this motion are clearly visible in the central horizontal plane as shown in Fig. 10-below. Here, in contrast to the $\mathrm{z}=0.005 \mathrm{~m}$ horizontal plane, a significant flow motion can be observed along the $y= \pm 0.3 \mathrm{~m}$ side walls. From the observed flow patterns, it is confirmed that the configuration with 3-magnets is a proper choice for studies of electromagnetic modulation of wall-boundary layers, while the 2-magnets configuration can be seen in light of an electromagnetically stirred flow - producing a low-pressure central region with an intensive swirling motion that can significantly affect underlying turbulent heat transfer mechanisms.

The character of the generated flow regimes is analysed from the time-series at characteristic monitoring points for different intensities of the applied DC currents. As expected, the highest intensity of the velocity fluctuations (turbulence) is observed in the proximity of the magnets where the Lorentz force is strongest. The characteristic time-evolutions and corresponding power spectral density (PSD) (in arbitrary units) ${ }^{2}$ of horizontal and vertical velocity components for different intensities of applied DC currents, at a monitoring point in the proximity of the centre of the lower wall, are shown in Fig. 11.

\footnotetext{
${ }^{2}$ The power spectra density (PSD) are evaluated by using ARMASA toolbox, Broersen (2007). Unlike the Fast Fourier Transformation (FFT) analysis, this method does not require any artificial smoothing of the row data in a time series.
} 
A weak electric current of $I=1$ A produces a transitional flow with a limited range of active scales and only a small part of the spectral density function exhibits a $5 / 3$ slope, see Fig. 11-below. In contrast, a strong electric current of $I=10$ A produces a PSD with a much wider range of active scales with a significant part of the spectrum following a $-5 / 3$ slope, indicating well developed turbulence. Very similar conclusions can be drawn from power spectral density distributions for the 3-magnets configuration, see Fig. 12. It can be seen that the monitored instantaneous velocity components exhibit less intermittent behaviour compared to the 2-magnets configuration. Again, a developed turbulence spectrum that follows a $-5 / 3$ slope is obtained for a stronger DC current of $I=10$ A. Also, by comparing the instantaneous velocity components and their spectra, for 2- and 3-magnets configurations, it can be concluded that the 2-magnets configuration is more efficient in generating a fully developed turbulent flow regime.

\section{Conclusions}

Combined numerical and experimental studies proved that the newly designed setup with different combinations of permanent magnets and electrodes can be used for fundamental studies of electromagnetically driven/affected flow, turbulence and heat transfer control. By changing the intensity of the imposed DC electric current, different flow regimes can be achieved. A particularly intriguing and interesting feature of the resulting flow is the simultaneous presence of different flow regimes in different parts of the setup.

A preliminary comparative assessment of PIV measurements and LES simulations with an electromagnetically extended subgrid closure showed good agreement for both 2- and 3-magnets configurations. The 3-magnet configuration demonstrated that it can be used for effective modulation of the wall-boundary layers. In contrast to such localised effects, the 2-magnets configuration can be used to generate an additional well-defined three-dimensional body-force that can put the entire liquid bulk into motion without any mechanical parts stirring. This is a very attractive feature for fundamental studies of interactions between fluid flow and electromagnetic fields. The results indicate also a great potential in using magnetic field for designing an efficient control of friction, heat and mass transfer in electrically conducting fluids. Future studies will address details of the second order statistics as well as non-isothermal situations. 


\section{Acknowledgement}

The research of S. K. has been made possible by a fellowship of the Royal Netherlands Academy of Arts and Sciences (KNAW). Mr. Andreas Wibowo is acknowledged for his assistance in performing the experiments. The high-performance computing facilities were provided by the NWO/NCF and SARA Computational and Network services, Amsterdam, The Netherlands. ${ }^{3}$

\section{References}

Akoun, G. and Yonnet, J., P., 1984. 3D analytical calculation of the forces exerted between two cuboidal magnets. IEEE Trans. Magnet. 20, pp.1962-1964.

Broersen, P. M. T., 2007. Let the Data Speak for Themselves. IEEE Transactions on Instrumentation and Measurement, Vol.56, No.3, pp. 814-823.

Cardoso, O., Marteau, D. and Tabeling, P., 1994. Quantitative experimental study of the free decay of quasi-2-dimensional turbulence. Phys. Rev. E 49 (1), pp. 454-461.

Hanjalić, K. and Kenjereš, S., 2000. Reorganisation of turbulence structure in magnetic RayleighBénard convection: a T-RANS study. Journal of Turbulence 1 (8), pp. 1-22.

Hanjalić, K. and Kenjereš, S., 2001. T-RANS simulation of deterministic eddy structure in flows driven by thermal buoyancy and Lorentz force. Flow, Turbulence and Combustion 66 (4), pp. 427-451.

Hanjalić, K. and Kenjereš, S., 2006. RANS-based VLES of thermal and magnetic convection at extreme conditions. Journal of Applied Mechanics - Transactions of the ASME 73(3), pp. 430-440.

Hennoch, C. and Stace, J., 1995. Experimental investigation of a salt water turbulent boundary layer modified by an applied streamwise magnetohydrodynamic body force. Phys. Fluids 7 (6), pp. 13711383.

Keane, R. D. and Adrian, R. J., 1990. Optimization of particle image velocimeters. Part I: Double pulsed systems. Meas. Sci. Tech. 1, pp. 1202-1215.

Kenjereš, S. and Hanjalić, K., 2000. On the implementation of effects of Lorentz force in turbulence

\footnotetext{
${ }^{3}$ www.sara.nl
} 
closure models. Int. J. Heat and Fluid Flow 21 (3), pp. 329-337.

Kenjereš, S. and Hanjalić, K., 2004. Numerical simulation of magnetic control of heat transfer in thermal convection. Int. J. Heat and Fluid Flow 25 (3), pp. 559-568.

Kenjereš, S., Hanjalić, K. and Bal, D., 2004. A direct-numerical-simulation-based second-moment closure for turbulent magnetohydrodynamic flows. Physics of Fluids 16 (5), pp.1229-1241.

Kenjereš, S., Hanjalić, K., Renaudier, S., Stefani, F., Gerbeth, G. and Gailitis, A., 2006. Coupled fluid-flow and magnetic-field simulation of the Riga dynamo experiment. Physics of Plasmas 13 (12), Art. No. 122308, pp.1-14.

Kenjereš, S. and Hanjalić, K., 2007a. Numerical simulation of a turbulent magnetic dynamo. Physical Review Letters 98 (10), Art. No. 104501, pp.1-4.

Kenjereš, S. and Hanjalić, K., 2007b. Numerical insights into magnetic dynamo action in a turbulent regime. New Journal of Physics 9, Art. No. 306, pp. 1-29.

Kenjereš, S., 2008. Electromagnetic enhancement of turbulent heat transfer. Physical Review E 78, Art. No. 066309, pp. 1-5.

Shepard, D., 1968. A two-dimensional interpolation function for irregularly-spaced data, In Proceedings of the 1968 23rd ACM national conference, ACM New York, New York, U.S.A.

Shimomura, Y., 1991. Large eddy simulation of magnetohydrodynamic turbulent channel flow under a uniform magnetic field, Physics of Fluids 3 (12), pp.3098-3106.

Thibault, J. P. and Rossi, L., 2003. Electromagnetic flow control: characteristic numbers and flow regimes of a wall-normal actuator, Journal of Physics D - Applied Physics 36 (20), pp.2559-2568.

Verdoold, J., Rossi, L. R. C., Tummers, M. J. and Hanjalić, K., 2003. Towards electromagnetic control of thermal convection. Proceedings of the ISFCMV Conference, Sorrento, Italy.

Verdoold, J., Witte, P., Hoek, L., Tummers, M. J. and Hanjalić, K., 2005. Identification and electromagnetic control of structures in Rayleigh-Bénard convection. In Proceedings of the 4th Symposium on Turbulence and Shear Flow Phenomena, TSFP-5, Virginia, U.S.A.

Verdoold, J., Tummers, M. J. and Hanjalić, K., 2006a. Oscillating large-scale circulation in turbulent Rayleigh-Bénard convection, Phys. Rev. E 73 (5), 056304. 
Verdoold, J., Wibowo, A. B., Tummers, M. J., Hanjalić, K., 2006b. Electromagnetic control of Rayleigh-Bénard convection, Proc. of the 5th Symposium on Turbulence, Heat and Mass Transfer, September 26-29, 2006, Dubrovnik, Croatia.

Verdoold, J., van Reeuwijk, M., Tummers, M. J., Jonker, H.J.J., Hanjalić, K., 2008. Spectral analysis of boundary layers in Rayleigh-Bénard convection, Phys. Rev. E 77 (1), 016303.

Rossi L., Vassilicos, J. C. and Hardalupas, Y., 2006a. Electromagnetically controlled multi-scale flows, J. Fluid Mech. 558, pp.207-242.

Rossi L., Vassilicos, J. C. and Hardalupas, Y., 2006b. Multiscale laminar flows with turbulentlike properties, Phys. Rev. Lett. 97 (14), 144501.

Yih, C. S., 2007. Tornado-like flows, Physics of Fluids 19, 076601. 


\section{Nomenclature}

$\begin{array}{ll}D & \text { characteristic dimension (m) } \\ R e=\frac{|\bar{U}|_{\text {max }} D}{\nu} & \text { Reynolds number (-) } \\ H a=B_{0} D \sqrt{\frac{\sigma}{\rho \nu}} & \text { Hartmann number (-) } \\ N_{M H D}=\frac{\sigma B_{0}^{2} D}{\rho|\bar{U}|_{\text {max }}} & \text { MHD Stuart (interactive) number (-) } \\ N_{E M H D}=\frac{f_{E M H D} D}{\rho|\bar{U}|_{\text {max }}^{2}} & \text { EMHD Stuart (interactive) number (-) } \\ I & \text { electric current intensity (A) } \\ f & \text { characteristic frequency (Hz) } \\ f_{E M H D} & \left.\text { volumetric electromagnetic force (N/m }{ }^{3}\right) \\ f^{\#} & \text { numerical aperture (-) } \\ \bar{P} & \text { resolved Pressure (Pa) } \\ \bar{U}_{i} & \text { resolved velocity (m/s) } \\ \mathbf{B} & \text { magnetic flux density (magnetic induction) (T) } \\ \mathbf{E} & \text { electric field intensity (V/m) } \\ \mathbf{F}^{\mathbf{L}} & \left.\text { Lorentz force (N/m }{ }^{3}\right) \\ \mathbf{J} & \left.\text { total current density (A/m }{ }^{2}\right)\end{array}$

\section{Greek symbols}

$\rho \quad$ fluid density $\left(\mathrm{kg} / \mathrm{m}^{3}\right)$

$\nu \quad$ kinematic viscosity $\left(\mathrm{m}^{2} / \mathrm{s}\right)$

$\nu_{t} \quad$ subgrid turbulence viscosity $\left(\mathrm{m}^{2} / \mathrm{s}\right)$

$\omega_{z}$ vertical vorticity $(1 / \mathrm{s})$

$\sigma \quad$ electric conductivity ( $\mathrm{S} / \mathrm{m}$ )

$\tau_{i j} \quad$ subgrid turbulent stress $\left(\mathrm{m}^{2} / \mathrm{s}^{2}\right)$

$\tau_{\theta i} \quad$ subgrid turbulent heat flux $(\mathrm{K} \mathrm{m} / \mathrm{s})$

$\Delta \quad$ grid cell size (m) 


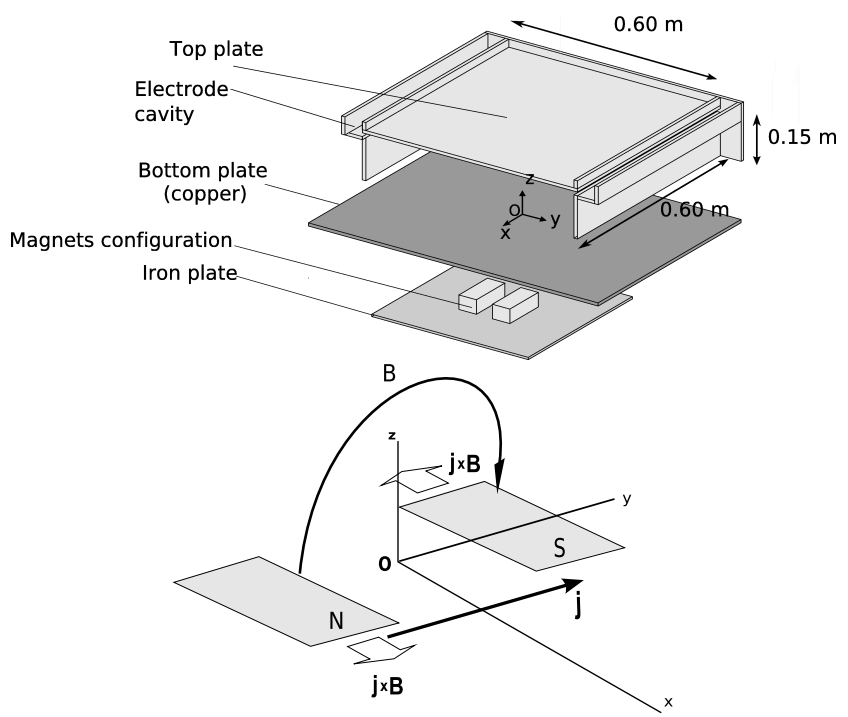

(b)

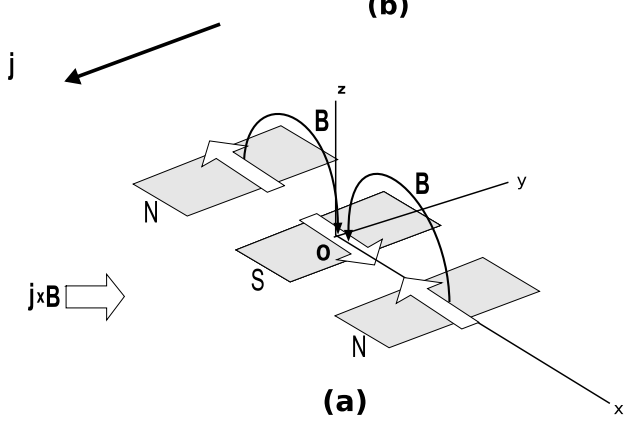

Figure 1: Above- Schematic of the experimental setup; Middle- "Shear" configuration with 2 magnets. Lower- "Pump in, pump out" configuration with 3 magnets. Directions of the imposed Lorentz force $(\mathbf{J} \times \mathbf{B})$ are indicated. 

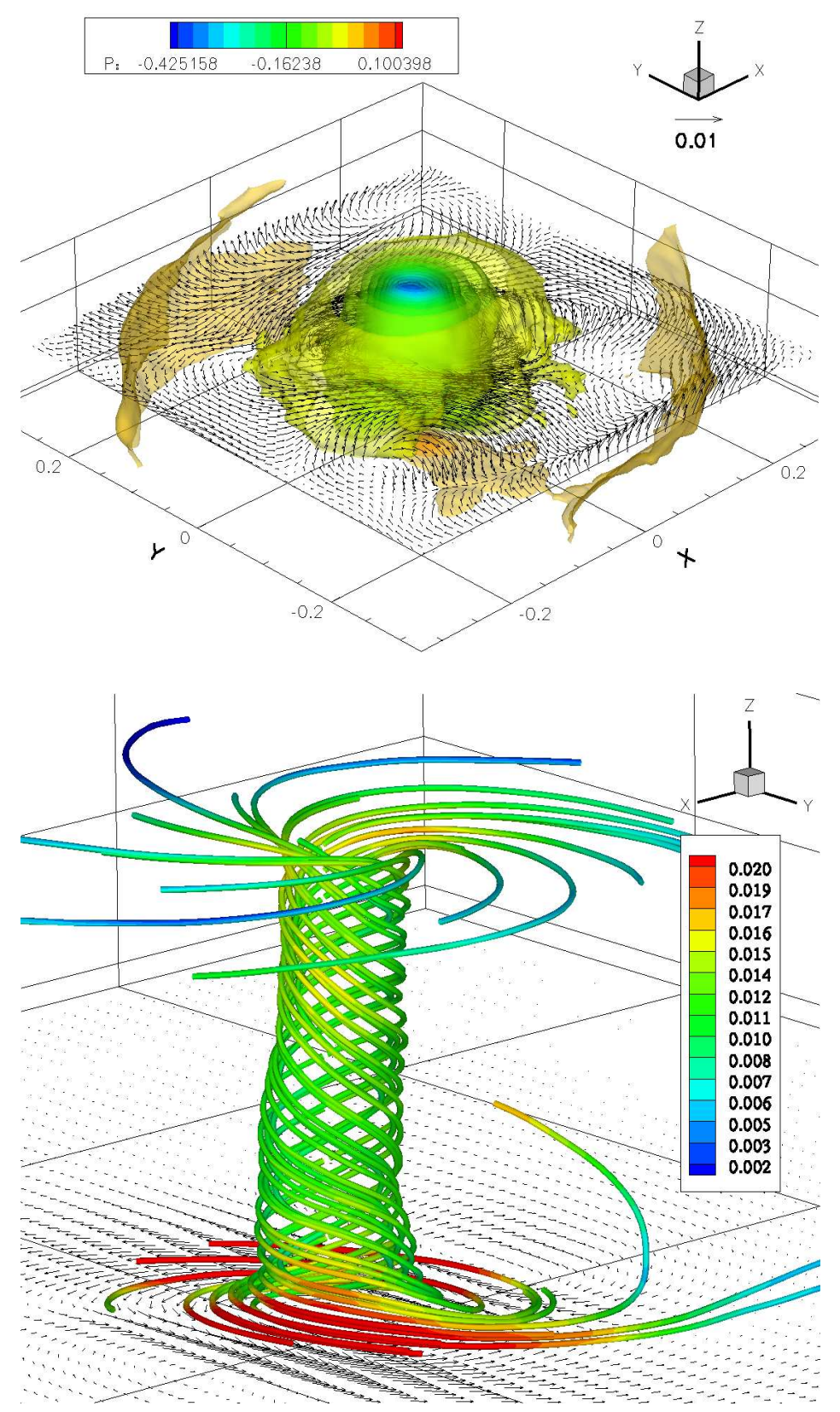

Figure 2: Visualisation of the flow for the 2 magnets configuration with $\left|B_{0}\right|=1 \mathrm{~T}$ and $I=10 \mathrm{~A}$ : the velocity vectors (in $\mathrm{m} / \mathrm{s}$ ) in the central horizontal plane with isosurfaces of low-pressure regions (in $\mathrm{Pa}$ ) (-above); zoom-in of the stream-traces coloured by the velocity magnitude (-below). 

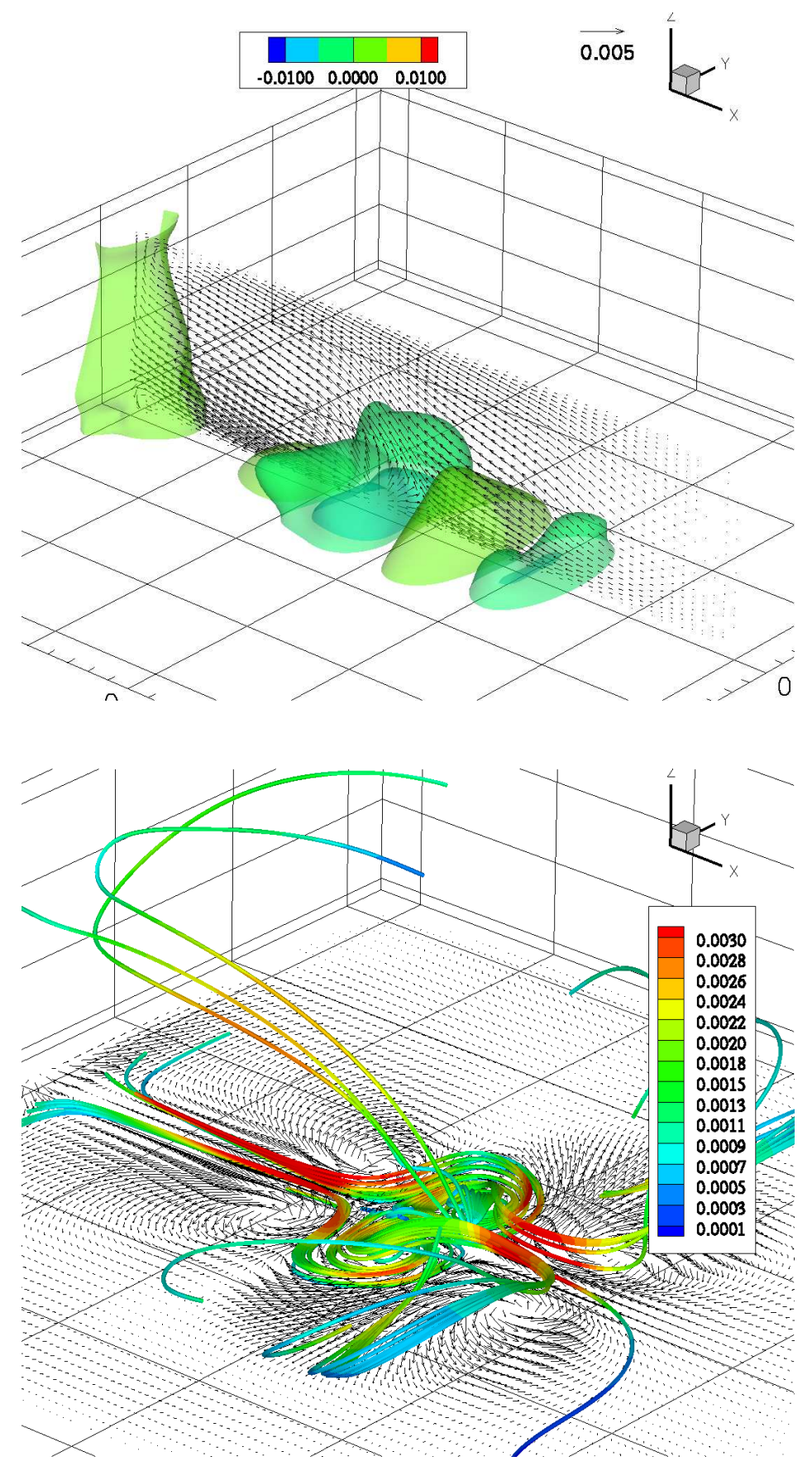

Figure 3: Visualisation of the flow for the 3 magnets configuration with $\left|B_{0}\right|=1 \mathrm{~T}$ and $I=0.5 \mathrm{~A}$ : the velocity vectors in the central vertical plane with isosurfaces of low-pressure regions (in Pa) (-above); zoom-in of the stream-traces coloured by the velocity magnitude (in $\mathrm{m} / \mathrm{s}$ ) (-below). 

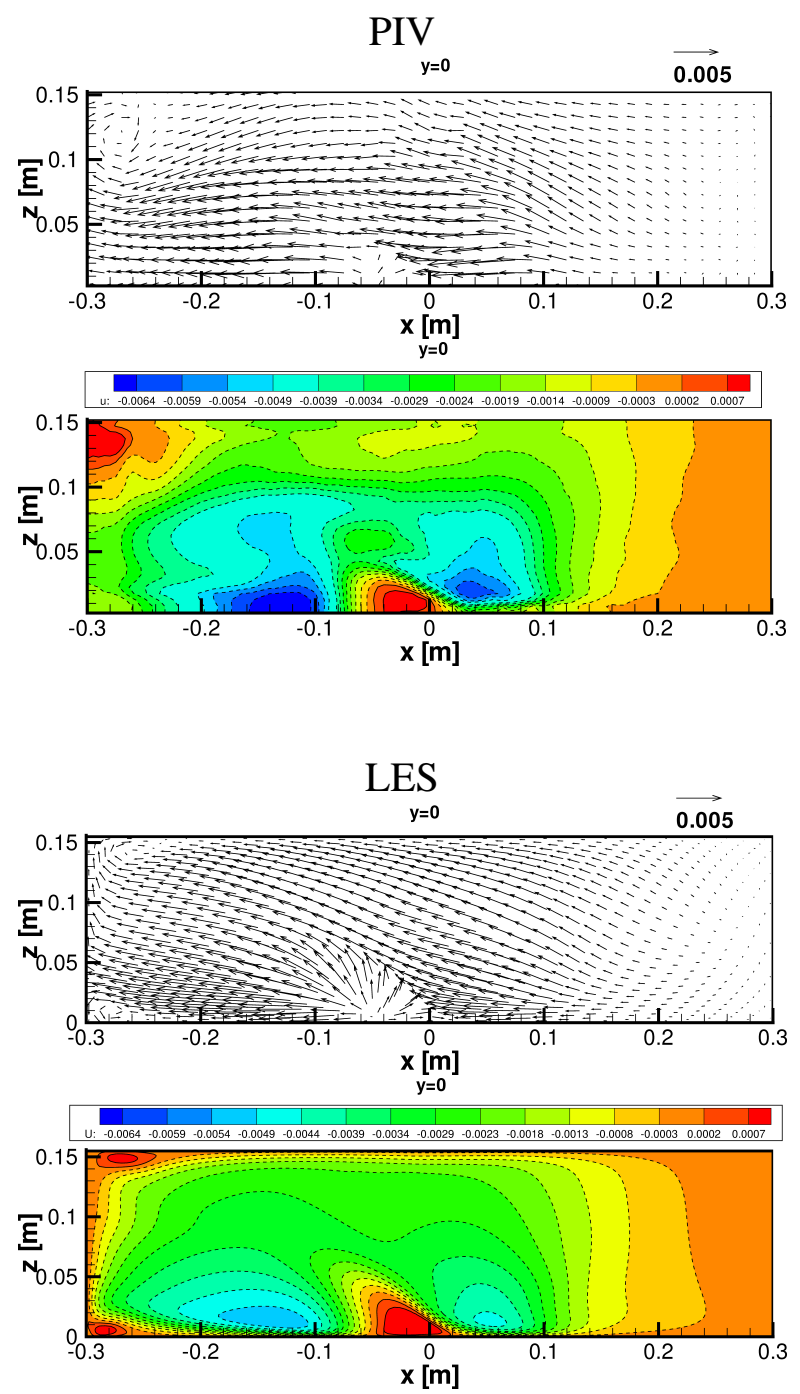

Figure 4: Comparison between PIV (-above) and LES (-below) results for the velocity vectors and contours of the horizontal velocity component (in $\mathrm{m} / \mathrm{s})$ in the central vertical plane $(y=0 \mathrm{~m})$. Configuration with 3-magnets $\left(\left|B_{0}\right|=1 \mathrm{~T}\right.$ and $\left.I=0.5 \mathrm{~A}\right)$. 


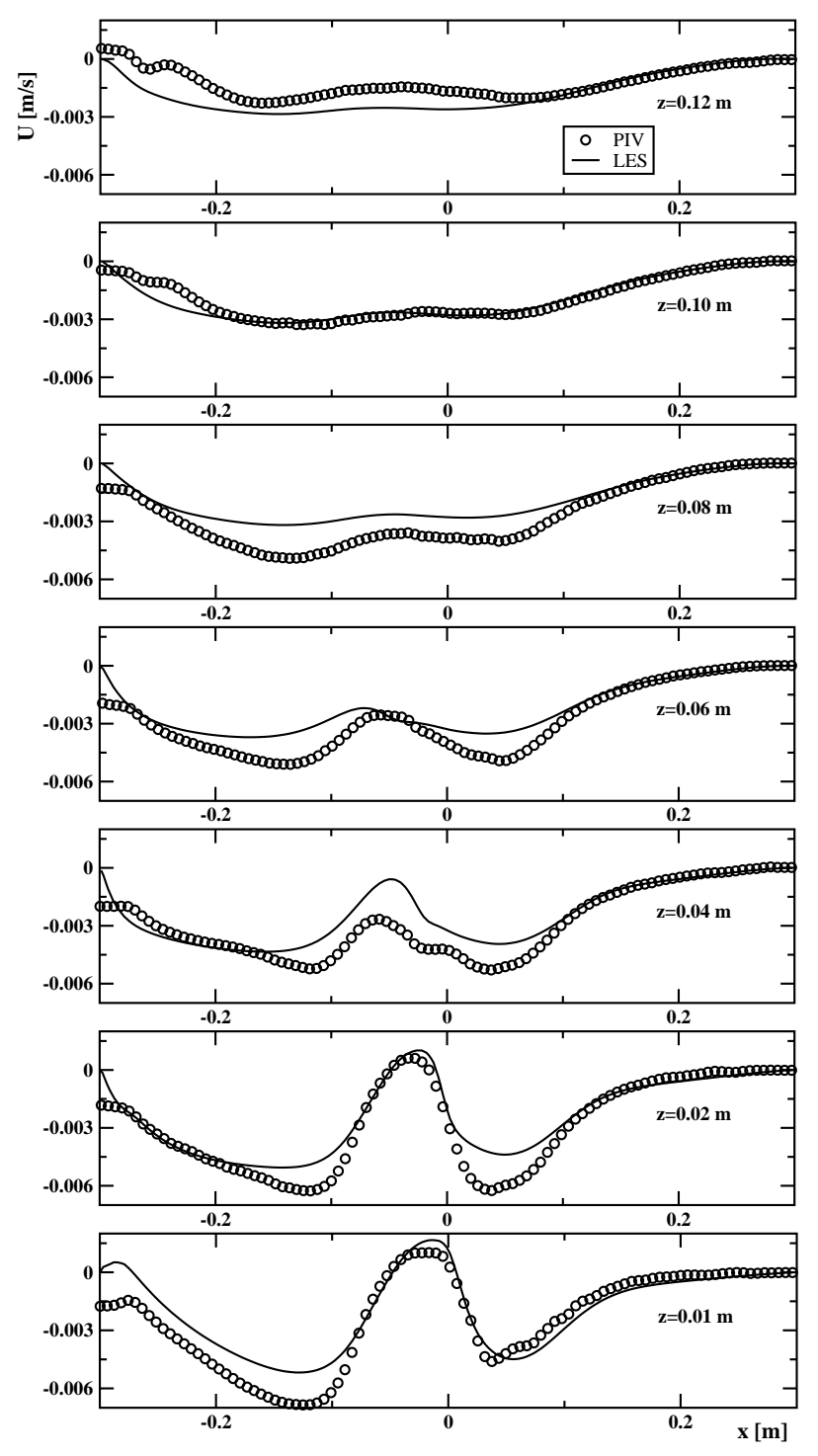

Figure 5: Horizontal velocity $(U)$ profiles at different vertical distances from the bottom wall. Comparison between PIV (o) and LES (-). Configuration with 3-magnets $\left(\left|B_{0}\right|=1 \mathrm{~T}\right.$ and $\left.I=0.5 \mathrm{~A}\right)$. 

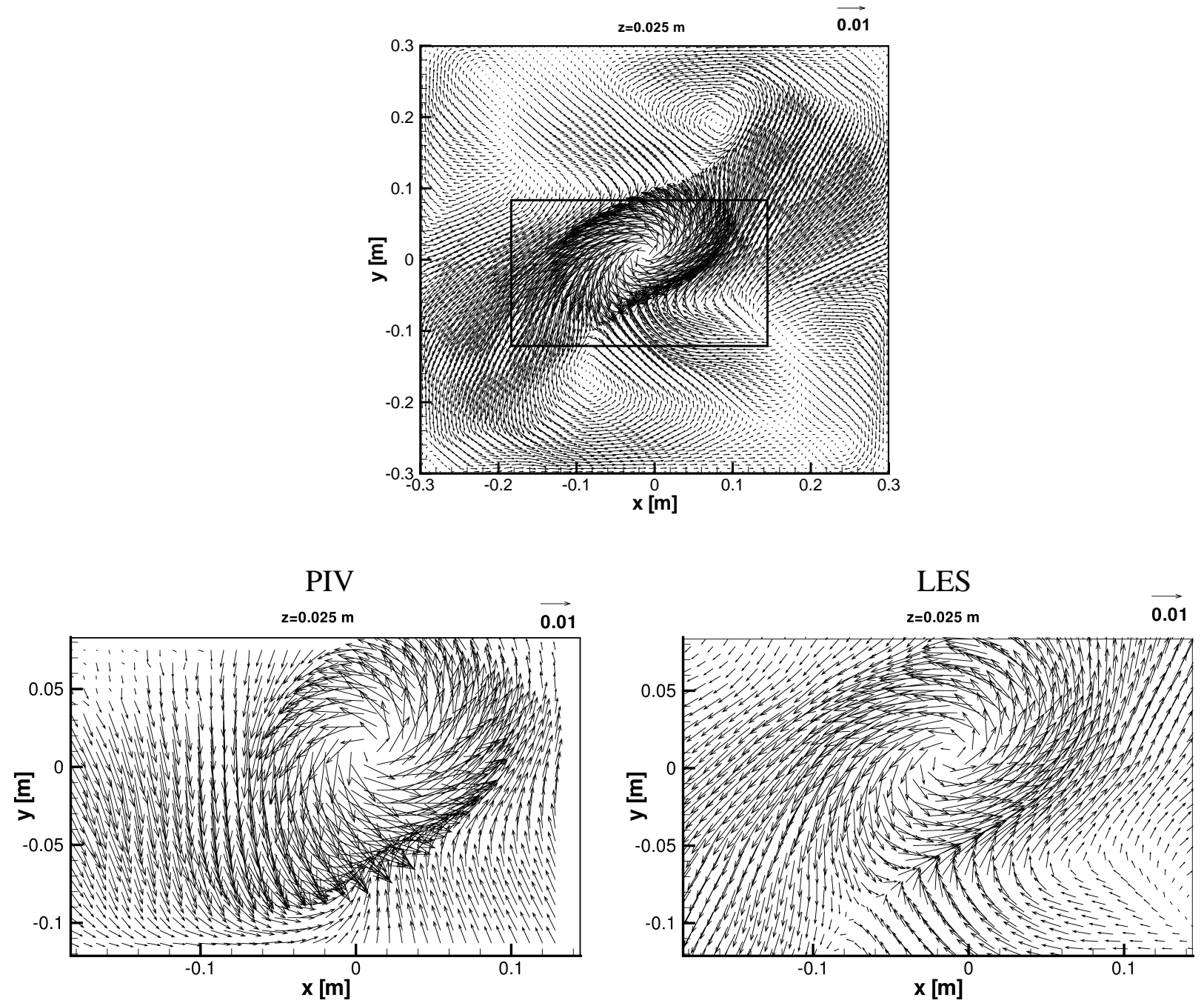

Figure 6: Velocity vectors in a horizontal plane at $z=0.025 \mathrm{~m}$ for the configuration with 2-permanent magnets $\left(\left|B_{0}\right|=1 \mathrm{~T}\right)$ and 2-electrodes $(I=10 \mathrm{~A})$. Above- LES velocities (in $\left.\mathrm{m} / \mathrm{s}\right)$ in the entire horizontal plane; Below- comparison between PIV (-left) and LES (-right) (zoom in of the marked region). 

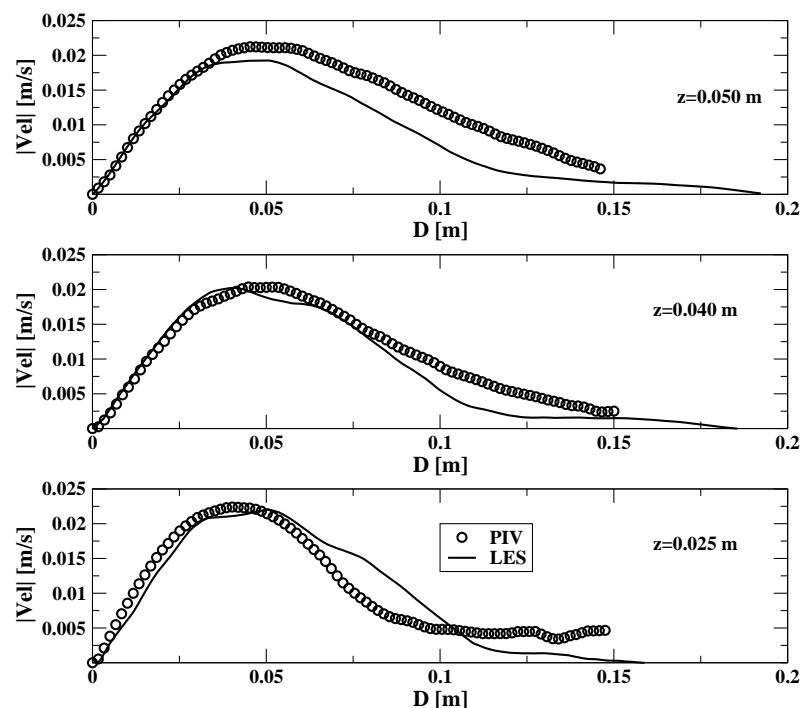

Figure 7: Comparison of the circumferentially averaged horizonal velocity magnitude $(|V|=$ $\left.\sqrt{U^{2}+V^{2}}\right)$ along the vortex diameter $\left(D=\sqrt{x^{2}+y^{2}}\right)$ at different vertical wall-distances. Configuration with 2-magnets $\left(\left|B_{0}\right|=1 \mathrm{~T}\right.$ and $\left.I=10 \mathrm{~A}\right)$.
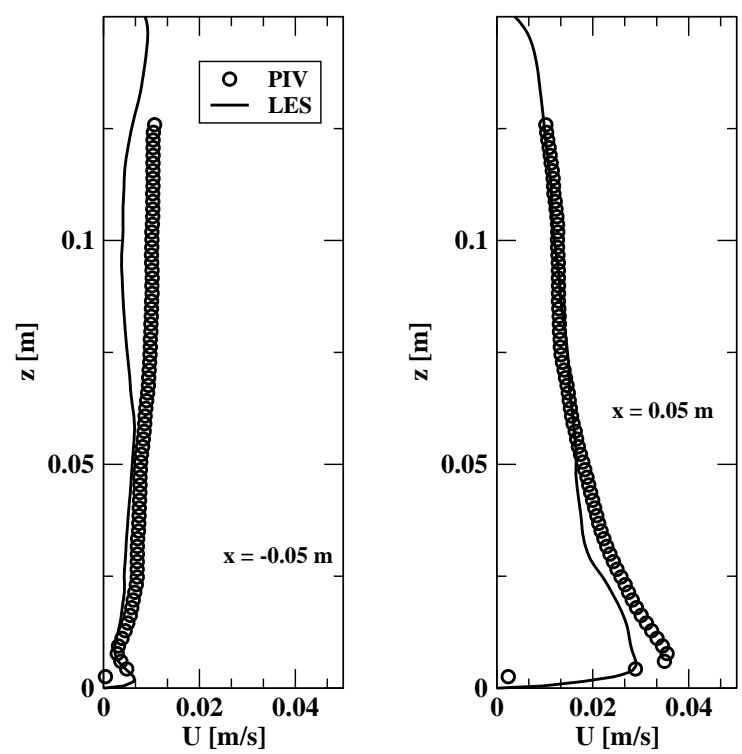

Figure 8: Vertical profiles of the horizontal velocity $(U)$ at two characteristic locations, $x=-0.05$ and $0.05 \mathrm{~m}$ in the vertical midplane $(\mathrm{y}=0 \mathrm{~m})$. Configuration with 2-magnets $\left(\left|B_{0}\right|=1 \mathrm{~T}\right.$ and $\left.I=10 \mathrm{~A}\right)$. 

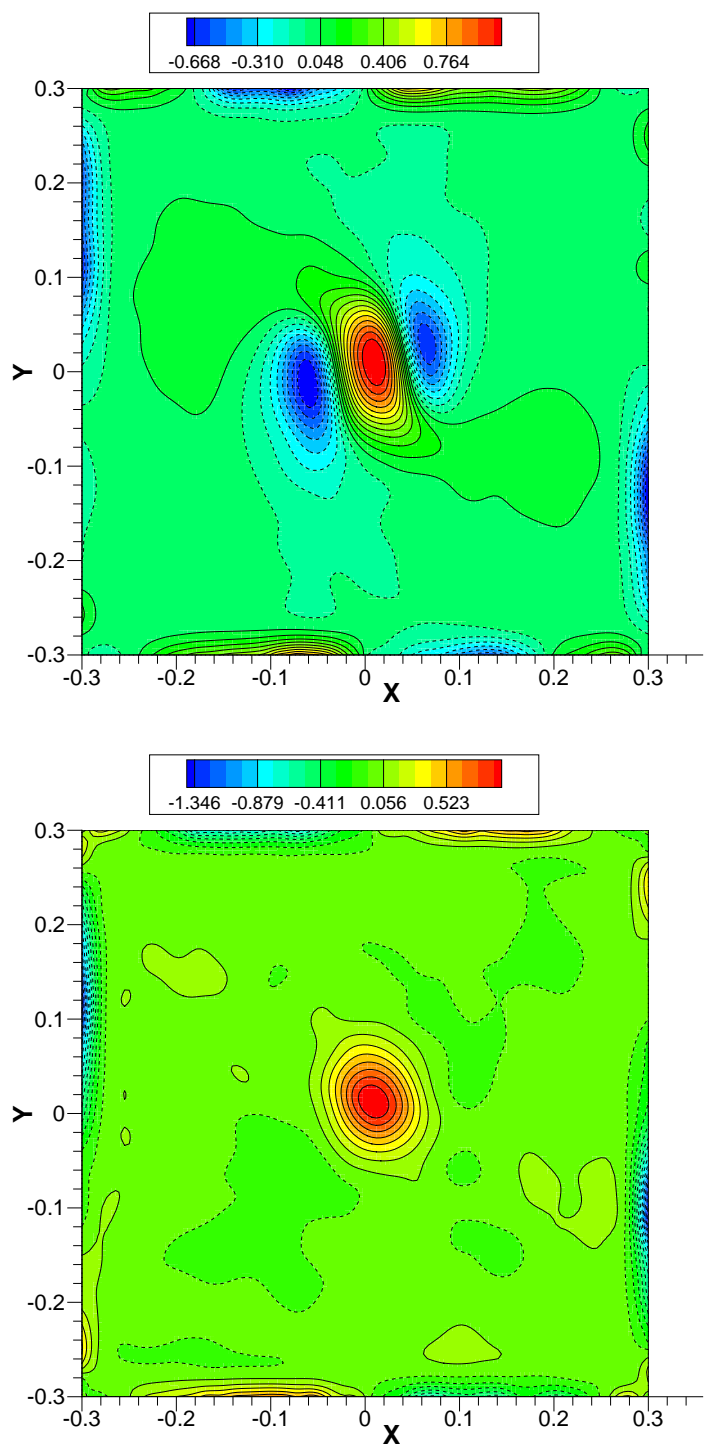

Figure 9: Vertical vorticity $\left(\omega_{z}\right)$ contours (in $1 / \mathrm{s}$ ) in the proximity of the bottom wall, $z=0.005 \mathrm{~m}$ (-above) and in the central horizontal plane, $z=0.075 \mathrm{~m}$ (-below), for the 2-magnets configuration, $\left|B_{0}\right|=1 \mathrm{~T}, I=10$ A. Results of a fully developed, time-averaged LES velocity field. 

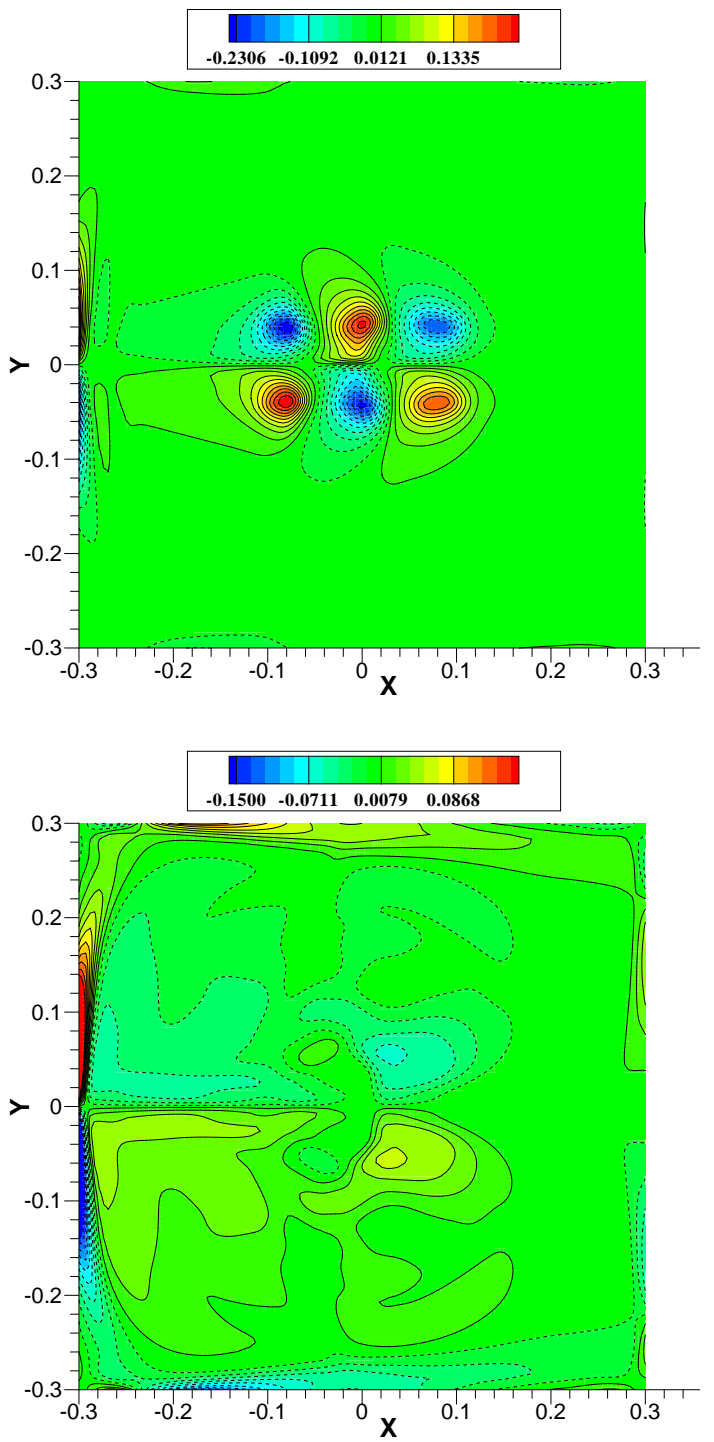

Figure 10: Same as Fig. 9 - now for the 3-magnets configuration, $\left|B_{0}\right|=1 \mathrm{~T}, I=0.5 \mathrm{~A}$. 

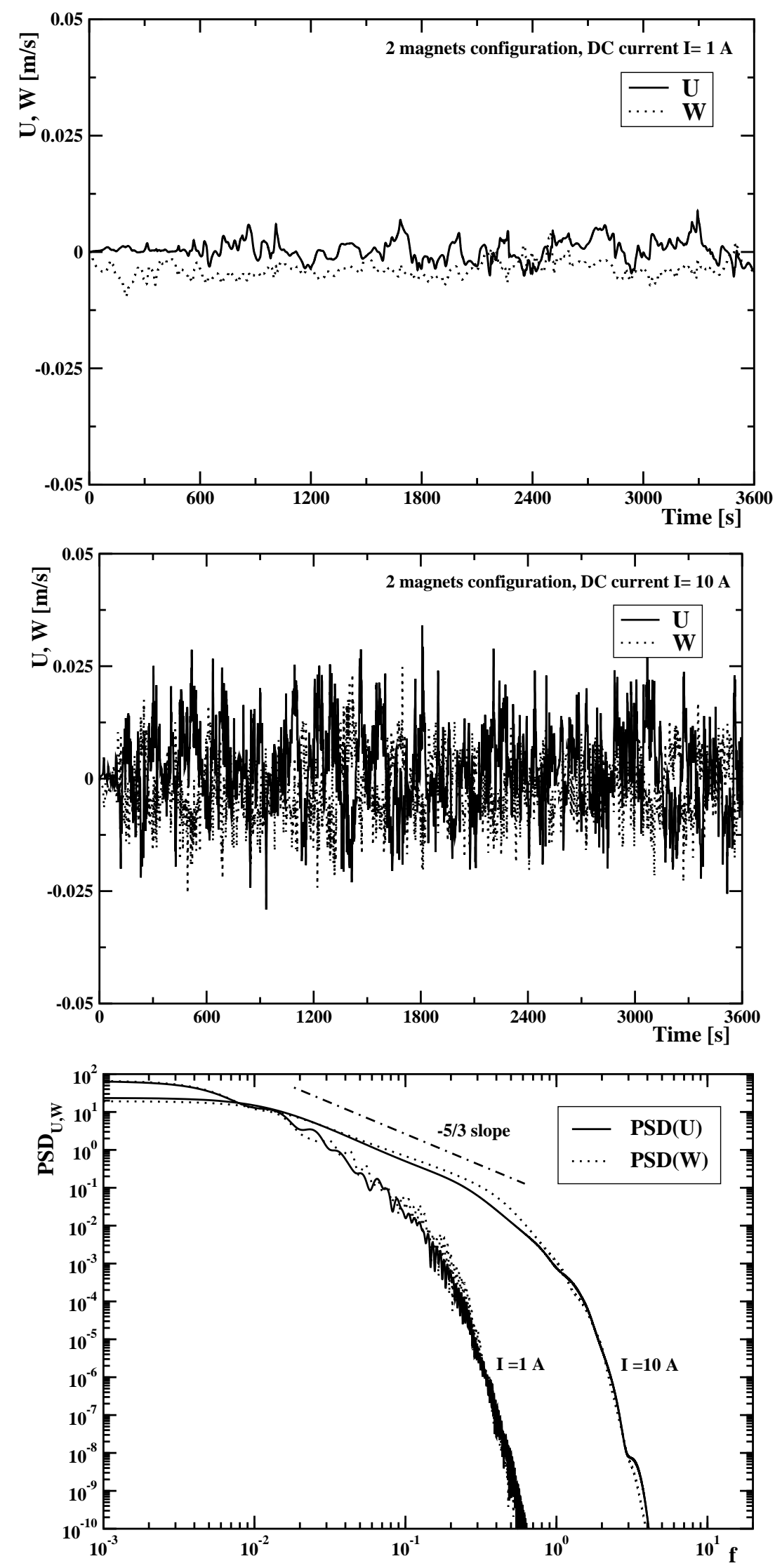

Figure 11: Time-evolution and power spectra density of the LES resolved velocity components (U, W) at a characteristic monitoring position $(\mathrm{x}=0 \mathrm{~m}, \mathrm{y}=0 \mathrm{~m}, \mathrm{z}=0.005 \mathrm{~m}): 2$ magnets configuration $\left|B_{0}\right|=1 \mathrm{~T}$, applied DC current of $I=1$ and 10 A. 

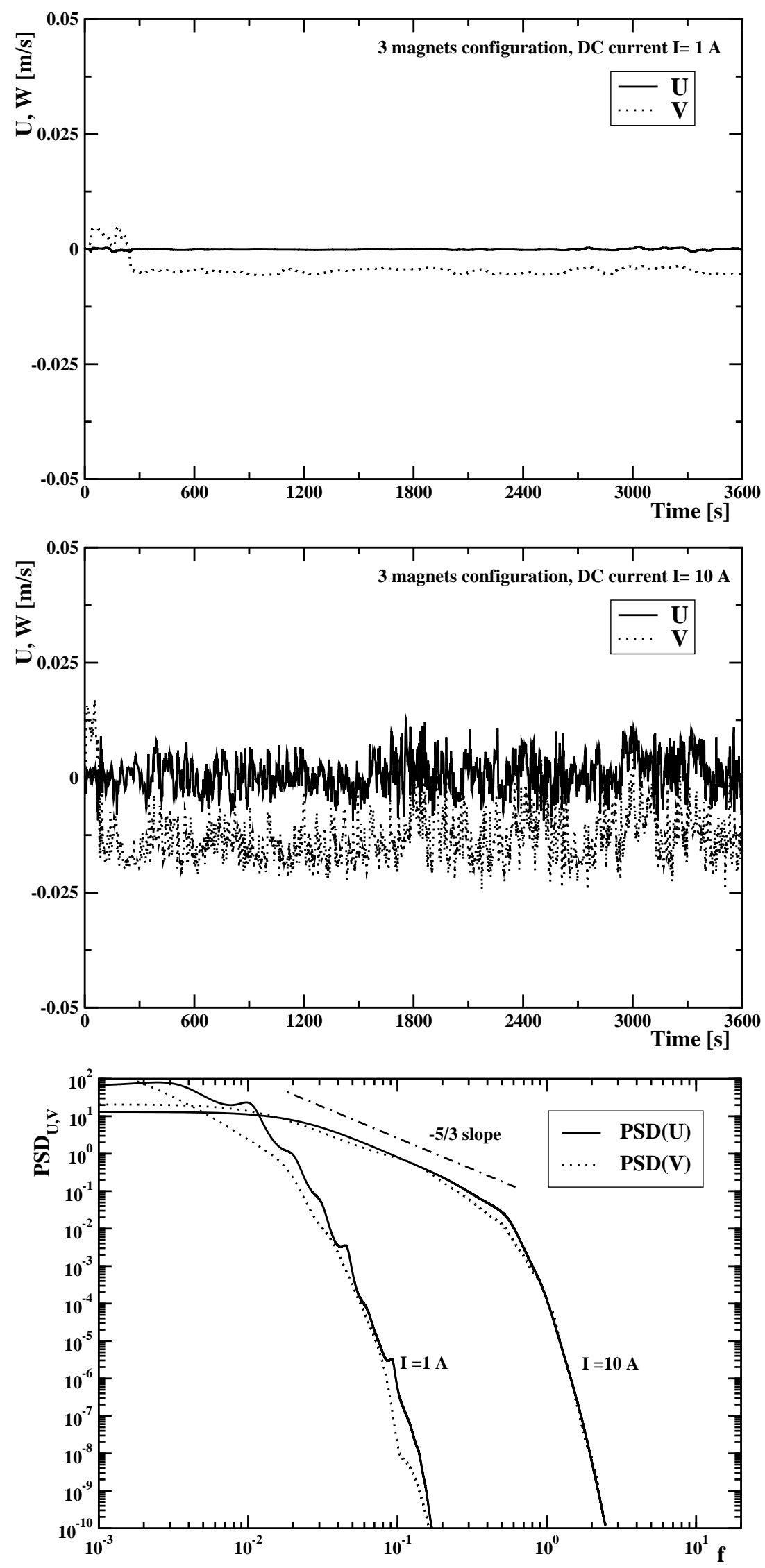

Figure 12: Time-evolution and power spectra density of the LES resolved velocity components (U, V) at a characteristic monitoring position $(\mathrm{x}=0 \mathrm{~m}, \mathrm{y}=0 \mathrm{~m}, \mathrm{z}=0.005 \mathrm{~m}): 3$ magnets configuration $\left|B_{0}\right|=1 \mathrm{~T}$, applied DC current of $I=1$ and 10 A. 